\title{
Why it is time to stop ostracizing social robots
}

To the Editor - With physical distancing and isolation measures deemed critical to slow the spread of COVID-19' ${ }^{1}$, social robots have finally found an opportunity to demonstrate their real value in society. These measures present important dilemmas. Isolation can cause a significant impact on psychological well-being. Additionally, physical distancing is not always possible, especially for healthcare personnel on the front-line, who consequently become exposed to a high risk of contagion ${ }^{2}$. Social robots could be key to solve these impasses.

The robotics community has repeatedly emphasized that robots could be useful to take action when humans cannot, especially in disaster situations that put human safety at risk. Yet there is still no implementation on a large scale. There are several reasons to explain the slow adoption. Robots, and particularly social robots, are generally considered expensive for their actual capabilities, and their cost-benefit tends to be disadvantageously compared to that of voice assistants. Additionally, there are sociocultural factors in Western societies that explain this reluctance when compared to Japan, for example. One might be robot aversion, influenced by science fiction, and arguably rooted in Judeo-Christian beliefs that associate the creation of 'human-like' creatures to an act of hubris ${ }^{3}$.
Social robots are able to interact with humans and in human physical environments with inherent immunity. This makes them particularly suitable for intervening in a situation that requires physical distancing and isolation, and should be considered for a fairer cost-benefit evaluation of the technology.

There are three strategic roles that social robots could fulfil to facilitate physical distancing as well as to reduce the drawbacks of isolation. First, to act as a liaison in tasks that require human-human interaction. This includes functions such as delivering medicines and supplies to patients in hospitals and monitoring them, dispensing hand sanitizer and masks in crowded places, robot avatars ${ }^{4}$ for doctors to assist patients, or even socially assistive self-driving ambulances. Second, to act as a safeguard to ensure contagion risk-free environments. This comprises surveillance and reminders of physical distancing in public spaces as well as environmental safety inspections and disinfection. Third, to act as well-being coaches by providing therapeutic and entertaining functions for quarantined patients.

Many of these functionalities have been occasionally tested during the pandemic ${ }^{5}$. Had the technology been ready earlier, social robots could have been decisive in minimizing intra-hospital transmissions or to palliate mental health issues resulting from quarantines and lockdowns, and not a mere anecdote of the pandemic.

It is time, more than ever, to let robots support us, rather than perceiving them as human-like creatures that pose a threat to humanity, to let them be the machines, so that we can be just humans.

\section{Laura Aymerich-Franch $\mathbb{}$}

Pompeu Fabra University, Barcelona, Spain.

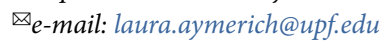

Published online: 29 June 2020

https://doi.org/10.1038/s42256-020-0202-5

References

1. Coronavirus Disease 2019 (COVID-19): Situation Report -72 (World Health Organization, 2020).

2. Rapid risk assessment: coronavirus disease 2019 (COVID-19) in the EU/EEA and the UK - eighth update. ECDC https://www. ecdc.europa.eu/en/publications-data/rapid-risk-assessmentcoronavirus-disease-2019-covid-19-pandemic-eighthupdate (2020).

3. Asimov, I. in Machines That Think: The Best Science Fiction Stories About Robots and Computers (eds. Asimov, I. \& Greenberg., P. S. W. and M. H.) Ch. 1 (Holt, Rinehart, and Winston, 1984).

4. Aymerich-Franch, L., Kishore, S. \& Slater, M. Int. J. Soc. Robot. 12, 217-226 (2020)

5. Coronavirus-fighting robots and drones. Reuters https://www. reuters.com/news/picture/coronavirus-fighting-robots-anddrones-idUSRTX7HZF6 (2020).

Acknowledgements

L.A.-F. is supported by the Ramón y Cajal Fellowship Program (ref. RYC-2016-19770), funded by Agencia Estatal de Investigación, Ministerio de Ciencia, Innovación y Universidades, and the European Social Fund. 